livraisons

d'Histoire

de l'Architecture

\section{Livraisons de l'histoire de l'architecture}

$35 \mid 2018$

Femmes, architecture et paysage

\title{
Les religieuses à l'époque moderne : architectes, gestionnaires de chantier et manouvrières
}

Nuns in the Modern Period: Architects, Site Managers and Laborers

Ordensfrauen in der Frühen Neuzeit: Architektinnen, Bauverwalterinnen, Arbeiterinnen am Bau

Julie Piront

\section{(2) OpenEdition}

Journals

Édition électronique

URL : http://journals.openedition.org/lha/915

DOI : 10.4000//ha. 915

ISSN : 1960-5994

Éditeur

Association Livraisons d'histoire de l'architecture - LHA

Édition imprimée

Date de publication : 15 juin 2018

Pagination : $29-40$

ISSN : 1627-4970

Référence électronique

Julie Piront, «Les religieuses à l'époque moderne : architectes, gestionnaires de chantier et

manouvrières », Livraisons de l'histoire de l'architecture [En ligne], 35 | 2018, mis en ligne le 15 juin 2020,

consulté le 26 janvier 2021. URL : http://journals.openedition.org//ha/915 ; DOI : https://doi.org/

$10.4000 /$ lha. 915 


\title{
LES RELIGIEUSES À L'ÉPOQUE MODERNE : ARCHITECTES, GESTIONNAIRES DE CHANTIER ET MANOUVRIÈRES
}

\begin{abstract}
"On a, depuis un siècle, contesté à dame Aldegonde Desmoulins l'honneur d'avoir dressé les plans, si pas de l'église, tout au moins de la façade de [l'abbatiale] Notre-Dame de la Paix [à Liège]. [...] Elle nous a laissé $[. .$.$] un monument sans prétention, de proportions$ modestes, mais à la fois grave, élégant et pur dans son style, aussi bien qu'approprié avec succès à sa destination multiple. Et ce monument, non point conçu, ni en tout ni en partie, mais complété par un autre artiste professionnel de mérite, le sculpteur Arnold du Honthoir, reste en somme la meilleure église, bâtie depuis le XVII siècle, dans ce quartier de Liège. L'honneur en soit désormais rendu et laissé, à l'humble et laborieuse architecte, fille de saint Benoît " ${ }^{1}$.
\end{abstract}

Au cours du XX $\mathrm{XX}^{\mathrm{e}}$ siècle, l'historiographie du monachisme occidental a pointé le rôle des moines ${ }^{2}$ et des religieux sur les chantiers de construction de leurs propres abbayes et monastères où ils assument les charges d'architectes, de bâtisseurs ou encore d'ingénieurs hydrauliciens. En Italie d'abord ${ }^{3}$, en France ensuite ${ }^{4}$, des chercheurs et chercheuses ont produit les monographies de quelques religieux archi-

1. Jules Demarteau, "L'église des Bénédictines de Liège. Son architecte Dame Aldegonde Desmoulins, poète wallon et miniaturiste (1640-1692), et son sculpteur Arnold du Honthoir ", Bulletin de l'Institut archéologique liégeois, t. 38, 1908, p. 198.

2. Voir, notamment, Anselme Dimier, Les Moines bâtisseurs. Architecture et vie monastique, Paris, Fayard, 1964, 223 p. ; Giovanni Coppola, «L'essor de la construction monastique en Normandie au XI ${ }^{\mathrm{e}}$ siècle : mécénat, matériaux et moines-architectes ", Annales de Normandie, vol. 42, nº 4, 1992, p. 335-349.

3. À titre d'exemples, citons Alfonso Gatto, Padre Andrea Martini : pittore, scultore, architetto, Roma, Bruni, 1986 ; Antonio Fernando Guida, Francesco da Copertino (1617-1692). Il frate cappuccino architetto del seminario di Matera, Rome, Edizioni universitarie romane, 2010 ; Richard Bösel et Lydia Salviucci Insolea (dir.), Mirabili Disinganni: Andrea Pozzo (Trento 1642-Vienna 1709) pittore e architetto gesuita, [Roma], Artemide, 2010, 319 p.

4. Parmi les travaux les plus récents, voir Philippe Bonnet, Les constructions de l'ordre de Prémontré en France aux XVII et $X V I I I^{e}$ siècles, coll. Bibliothèque de la Société française d'archéologie, 15, Paris, Arts et métiers graphiques, 1983, p. 28-35; Erwann Le Franc, "Les religieux architectes en Bretagne au XVII ${ }^{\mathrm{e}}$ siècle ", Bulletin et mémoires de la société polymathique du Morbihan, 2014 , t. 140, p. 423-456; Adriana Sénard, Étienne Martellange (1569-1641) : un architecte "visiteur" de la Compagnie de Jésus à travers la France au temps de Henri IV et de Louis XIII, thèse de doctorat inédite, 5 vol., Université de Toulouse Le Mirail et Université Paris-Sorbonne, 2015, 1415 p. 
tectes à l'époque moderne. L'intérêt pour les religieuses est beaucoup plus récent, même s'il remonte déjà à une trentaine d'années. Plusieurs chercheurs, surtout italiens et anglo-saxons ${ }^{5}$, ont montré combien les pratiques artistiques dans les couvents féminins, notamment à l'époque moderne, se révèlent diversifiées, sortant de l'anonymat des religieuses peintres, miniaturistes, doreuses, brodeuses, orfevres, dessinatrices, dentellières, compositrices et musiciennes, mais aussi des architectes, des gestionnaires de leurs chantiers et même des manouvrières (ill. 1) ${ }^{6}$. Si l'œuvre architecturale des visitandines a été révélée par Laurent Lecomte $^{7}$, celle des religieuses des autres congrégations reste encore largement méconnue malgré l'article fondateur de Philippe Bonnet ${ }^{8}$.

Si les compétences en matière d'architecture et de gestion de chantier des religieuses aux cours des Temps Modernes sont encore peu abordées par l'historiographie, ce constat peut s'expliquer d'une part par la nature des sources écrites qui permettent d'identifier leurs interventions. Elles consistent en biographies de religieuses, en récits de fondations des monastères, en chroniques ou annales. Ces textes, rédigés sous forme manuscrite par les communautés au cours de leur histoire, sont avant tout destinés à un usage interne et demeurent pour la plupart inédits. Souvent largement postérieurs aux faits, ils consistent en une réécriture du passé et sont généralement destinés à nourrir la mémoire de la communauté et à l'aider à construire son identité autour de religieuses aux vertus méritantes, dignes de servir de modèle aux autres membres de la communauté, présentes et à venir. Ces sources s'avèrent particulièrement intéressantes, car elles présentent ces femmes comme des personnalités dotées des vertus spirituelles les plus admirables, mais aussi remarquables par leurs talents d'architectes, d'entrepreneuses et de gestionnaires. Si ces textes doivent donc être étudiés avec les précautions critiques qui s'imposent, ils sont incontournables pour l'étude des religieuses architectes.

Les religieuses, soumises à une clôture plus rigoureuse que leurs homologues masculins, ne conçoivent et ne construisent généralement qu'un seul édifice au cours de leur vie: leur propre monastère. La production architecturale de ces femmes

5. Voir Jeffrey F. Hamburger, Nuns as artists. The visual culture of a medieval convent, BerkeleyLos Angeles-Londres, University of California Press, 1997, 342 p. et les différentes contributions publiées dans le collectif de Sheila Barker et Luciano Cinelli (dir.), Artiste nel Chiostro : Produzione artistica nei monasteri femminili in età moderna, Memorie Domenicane, nouv. série, vol. 46, 2015, $691 \mathrm{p}$.

6. Je remercie Adriana Sénard de m'avoir signalé l'existence de cette image.

7. Laurent Lecomte, "Jeanne de Chantal, maître de l'ouvrage de son ordre ", dans Bernard Dompnier et Dominique Julia (dir.), Visitation et visitandines aux XVII et XVIII $I^{e}$ siècles, actes du colloque d'Annecy (3-5 juin 1999), Saint-Étienne, Presses Universitaires de Saint-Étienne, 2001, p. 89-107 ; Laurent Lecomte, "De la manœuvre au maître d'œuvre: les visitandines sur les chantiers ", dans Gérard Picaut et Jean Foisselon (dir.), De l'ombre à la lumière : art et histoire à la Visitation 16102010, actes du colloque (Moulins, musée de la Visitation, 22-23 octobre 2010), Paris/Moulins, Somogy/Musée de la Visitation, 2011, p. 247-255; Laurent Lecomte, Religieuses dans la ville. L'architecture des visitandines (XVII ${ }^{e}$-XVIII ${ }^{e}$ siècles), Paris, 2013, p. 102-106.

8. Philippe Bonnet, "La pratique des arts dans les couvents de femmes au XVII ${ }^{\mathrm{e}}$ siècle ", Bibliothèque de l'école des chartes, t. 147, 1989, p. 433-472. 


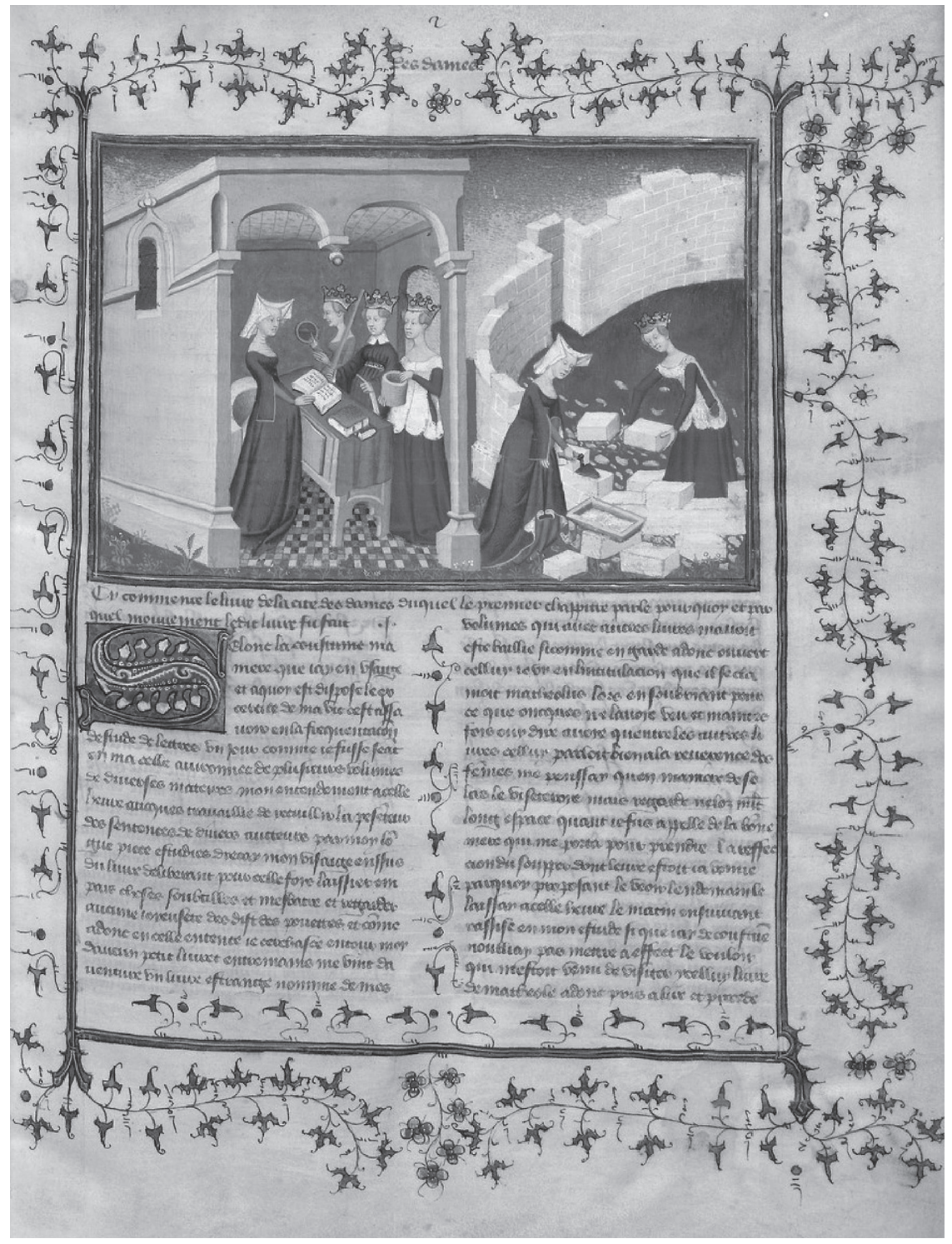

Ill. 1 : Maître de la Cité des Dames, Femmes construisant la muraille de la "Cité des Dames ", 14001410, extrait de Christine de Pizan, Le livre de la Cité des Dames, XV siècle. Bibliothèque nationale de France, département des manuscrits, Français 607, f. 2r. (C) Cliché BnF, département des manuscrits. 
reste donc moins abondante que celles des religieux architectes qui mènent fréquemment des carrières itinérantes ${ }^{9}$. Il existe bien sûr des exceptions, à l'exemple de la mère Madeleine-Élisabeth de Chaumont. Devenue prieure des visitandines de Vannes en 1668, elle est élue à la tête des couvents de Chartres huit ans plus tard, puis de Compiègne en 1682, ayant été choisie précisément pour ses talents d'architecte $^{10}$. En une vingtaine d'années, elle aura contribué à l'édification des trois monastères dont elle a eu la charge.

La Réforme catholique est propice à l'émergence de nombreux nouveaux ordres féminins. Qu'il s'agisse de créations ou de réformes de congrégations plus anciennes, tous sont soumis à la clôture stricte, un point de discipline réaffirmé avec fermeté par les décrets du concile de Trente $(1545-1563)^{11}$. Au sein de cette multitude de projets religieux, les archives de plusieurs ordres ont révélé la présence de religieuses architectes. C'est le cas notamment des carmélites déchaussées issues de la réforme du Carmel entreprise par Thérèse d'Avila (1515-1582) en 1567. Tout aussi célèbres, les ursulines, congrégation enseignante, se structurent en deux branches, l'une fondée en Italie en 1535 par Angela Merici (1474-1540), l'autre établie en FrancheComté à l'initiative d'Anne de Xainctonge (1567-1621) en 1606. Les visitandines sont, quant à elles, fondées à Annecy en 1610 par Jeanne de Chantal et François de Sales, évêque de Genêve. À ces ordres, on peut encore en ajouter deux autres, bien moins connus : les annonciades célestes, fondées à Gênes en 1604 par Vittoria Fornari et les bénédictines de la Paix Notre-Dame, réformées la même année à Douai par Florence de Werquignoel (1559-1638).

\section{Des religieuses architectes}

Dès les premières années qui suivent sa fondation, chaque communauté doit s'atteler à la conception puis à l'édification de bâtiments monastiques et d'une église à la fois conformes aux besoins du groupe et à la clôture stricte des religieuses, et adaptés aux contraintes du parcellaire urbain. Les ordres religieux féminins les plus centralisés disposent d'un "plan-type » théorique qui leur sert de guide. Ces plans circulent d'abord sous forme manuscrite avant d'être imprimés et diffusés par le biais des cérémoniaux qui visent à uniformiser les pratiques et le déroulement des cérémonies qui rythment le quotidien des religieuses, ce qui passe par une attention accordée aussi aux espaces liturgiques et au cadre architectural. La référence à un

9. Il suffit d'examiner la production architecturale et le parcours du jésuite Étienne Martellange. Voir Adriana Sénard, Étienne Martellange (1569-1641), op. cit.

10. Paris, Bibliothèque Mazarine, ms 2436: Histoire chronologique des fondations de tout l'Ordre de la Visitation de Sainte-Marie, institué par saint François de Sales, évêque et prince de Genève, et par la Vénérable Mère Jeanne-Françoise Frémiot, baronne de Chantal, première supérieure de l'Ordre, vol. 7 , $\mathrm{XVIII}^{\mathrm{e}}$ siècle, p. 150-151; BnF, Collection de lettres circulaires émanées des religieuses de la Visitation Sainte-Marie des différents monastères de France ou à elles adressées de France ou de l'étranger, t. 123.

11. Giuseppe Albergio (dir.), Le Magistère de l'Église. Les Conciles cecuméniques, t. II-2. Les décrets : De Trente à Vatican II, éd. française dirigée par André Duval et al., Paris, Cerf, 1994, p. 1581. 
modèle contribue aussi à la construction identitaire de ces ordres, un souci qui se manifeste dans un contexte de prolifération des projets de vie religieuse féminine dans les villes de la catholicité ${ }^{12}$. Les visitandines ${ }^{13}$ et les carmélites ${ }^{14}$ bénéficient ainsi de plans directeurs (ill. 2). La sœur Marie de la Sainte Trinité (1579-1647), première professe française de l'ordre et fondatrice des deux couvents de Troyes, "dressa elle-même le plan du monastère sur le modèle de ceux d'Espagne » ${ }^{15}$, manifestant une volonté de se référer à une norme, qu'il s'agisse d'un plan idéal ou, comme c'est le cas ici, du plan des premières maisons de l'ordre. Les annonciades célestes, elles aussi, se sont tournées vers leurs «mères de Gênes ", fondées par Vittoria Fornari, pour obtenir les plans de leur bâtiment ${ }^{16}$.

Sur place, la conception du plan du nouveau monastère peut-être prise en charge par les fondatrices qui l'élaborent alors en groupe après l'acquisition d'une ou de plusieurs parcelles mitoyennes. À Joinville (act. département de la Haute-Marne) comme à Liège, ce dessin sert de plan-directeur jusqu'à l'achèvement des travaux de construction qui s'échelonnent généralement durant plusieurs décennies, au rythme des rentrées financières de la communauté ${ }^{17}$. Dans d'autres cas, une religieuse entreprend seule de faire un plan : d'après les sources consultées, son projet est systématiquement soumis à l'approbation de personnes extérieures à la communauté. Ainsi, au milieu du XVII ${ }^{e}$ siècle, la prieure des annonciades célestes de Namur dresse, à la demande de l'évêque, " un plant en quarton [...] avec estonnement de ceux qui l'on veu, et d'elle mesme ${ }^{18}$. Cette manière de présenter les choses prend ici une tournure presque miraculeuse : tout le monde s'étonne, y compris la religieuse qui témoigne par là d'une grande modestie. Outre cette vertu morale, la religieuse fait preuve d'une autre qualité, l'obéissance au supérieur, puisqu'elle s'attèle à cette tâche à la demande de l'évêque ; modestie et obéissance, deux valeurs qui caractérisent la

12. Marie-Élisabeth Henneau, «Notion d'identité(s) et monde régulier. Quelques réflexions au regard d'un ordre de contemplatives à l'époque moderne ", Trajecta, vol. 18, 2009-3, p. 195-208.

13. Le premier plan-type imprimé est diffusé dans le Coustumier et Directoire pour les sours religieuses de la Visitation Saincte Marie, Lyon, Vincent de Cœursilly, 1628. Voir à ce sujet, Laurent Lecomte, "Jeanne de Chantal, maître de l'ouvrage de son ordre", op. cit., p. 90-93.

14. Cérémonial pour l'usage des religieuses carmélites deschaussées de l'ordre de Nostre Dame du Mont Carmel érigé en France selon la première Règle, Paris, G. Josse, 1659.

15. Chroniques de l'ordre des carmélites de la réforme de Sainte Thérèse depuis leur introduction en France, t. 3, Troyes, impr. Anner-André, 1856, p. 426.

16. Julie Piront, "Copies et modèles architecturaux aux frontières de la catholicité : les sources d'inspiration des monastères des annonciades célestes (XVII - XVIII ${ }^{\mathrm{e}}$ siècles) ", dans Marie-Élisabeth Henneau, Corinne Marchal et Julie Piront (dir.), Amazones de Dieu. Profils, engagements et réalisations de femmes entre Gênes et Liège (XI $-X V I I I^{e}$ s.), actes des trois journées et du colloque de l'ANR Lodocat (axe 1), Paris, Garnier, à paraître.

17. Langres, Dépôt d'Art sacré, Annales du Monastère de Notre Dame de l'Annonciade Céleste de Joinville, mises en ordre l'année 1698, XVII ${ }^{\mathrm{e}}$ XVIII ${ }^{\mathrm{e}}$ siècle, p. 46 ; Bibliothèque Royale de Bruxelles, ms $\mathrm{n}^{\circ} 19612$ : Sœur Marie Françoise Augustine Joseph [Laloire], Histoire de l'établissement de l'ordre de l'annonciade céleste dans la ville de Liège, [1746-1747], p. 260-261.

18. San Cipriano, archives des annonciades célestes de Gênes, Fondations, 37 : Abrégé de la fondation de notre monastere de l'Anonciade ditte celeste de la ville de Namur faict par les Rdes Mères de Nancy l'an 1630, [XVII ${ }^{\mathrm{e}}$ siècle ?], non paginé. 


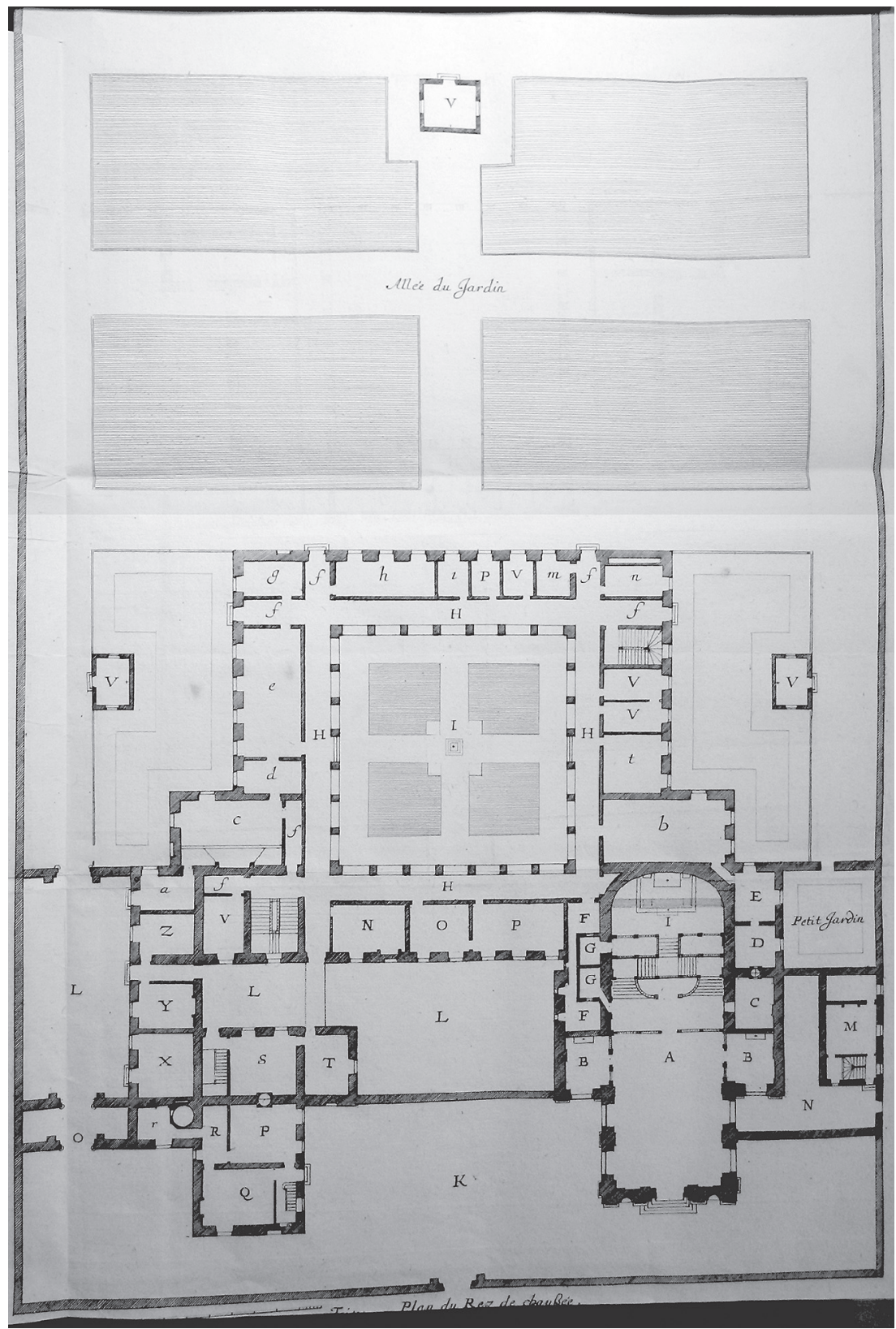

Ill. 2 : Plan-type du rez-de-chaussée du Carmel, XVII e siècle, extrait du Cérémonial pour l'usage des Carmélites deschaussées de l'Ordre de Nostre Dame du Mont Carmel érigé en France selon la première Règle. Composé conformément aux usages de l'Ordre, par le Révérend Père Dom Augustin Galice, Général des Révérends Pères de la Congrégation de S. Paul, dits Bernabites, à la prière des Supérieurs desdites Religieuses, Paris, G. Josse, 1659. (C) Cliché Julie Piront. 
parfaite religieuse au XVII ${ }^{\mathrm{e}}$ siècle ${ }^{19}$. Mais qui sont les personnes qui ont vu ce plan ? L'évêque et sans doute aussi des architectes professionnels, comme ce fut le cas chez les annonciades célestes de Besançon qui décident en 1732 d'entreprendre une nouvelle construction. La prieure sollicite une des religieuses de sa communauté qui se distingue des autres par "de l'intelligence et du gout pour l'architecture »: son projet est ensuite légèrement modifié par l'architecte (Jean-Pierre ?) Galezot ${ }^{20}$.

Cette religieuse de Besançon, demeurée anonyme, semble avoir eu des prédispositions à se voir confier le projet du nouveau bâtiment. Peut-être y a-t-elle été formée ? Malheureusement, la formation des religieuses architectes demeure souvent méconnue, faute de sources. Néanmoins, plusieurs indices permettent de dégager différents canaux d'apprentissage. D'une part, quelques religieuses semblent avoir eu accès aux traités théoriques et aux manuels d'architecture. C'est le cas notamment d'une ursuline d'Amiens, Françoise Becquerel de Sainte-Marie-des-Anges (1618-1689), qui aurait " appris, par la seule lecture des livres, la perspective, l'architecture, la géométrie " ${ }^{21}$, des connaissances qui définissent l'architecte sous l'Ancien Régime ${ }^{22}$. Une autre, visitandine au monastère de Mamers, Marie-Aymée Davoust (1633-1697), « sçavoit les règles de la musique, l'arithmétique et l'architecture, qui fut fort utile pendant le bâtiment " ${ }^{23}$.

D'autre part, certaines religieuses ont eu la possibilité de se déplacer d'un couvent à l'autre afin d'examiner sur place les réalisations les plus récentes en matière de bâtiments monastiques, comme en témoigne une lettre de l'évêque du Mans, Pierre Rogier du Crévy, en 1713 :

Connaissans par nous-même le besoin qu'a votre communauté de se bastir au plus tôt $[\ldots]$, si vous allez visiter les monastères nouvellement construits dans les maisons de votre institut, nous vous ordonnons de vous transporter dans ceux qui vous seront indiqués par notre chère fille votre supérieure $[\ldots]^{24}$.

19. Marco Aurelio Grattarola, Practique de la vie spirituelle pour les religieuses et autres personnes désireuses de s'advancer à la perfection chrestienne, trad. française par Andrieu Curia, Douai, Jean Bogart, 1623, p. 331-347 et 362-365.

20. Langres, Dépôt d'Art sacré : Livre contenant l'establissement de nostre monastère de l'annonciade, fait en cette cité de Besançon au mois de mars de l'an 1648, ensemble les choses plus remarquables qui y sont arrivées du depuis, $\mathrm{XVII}^{\mathrm{e}}-\mathrm{XVIII}{ }^{\mathrm{e}}$ siècles, non paginé.

21. Paris, bibliothèque de la Sorbonne, ms 769 : Matériaux pour un nécrologe des ursulines. Recueil de lettres circulaires imprimées, adressées à différents monastères et notes manuscrites, $\mathrm{XVII}{ }^{\mathrm{e}}$ siècle.

22. L'architecte est «celui qui donne le dessein des ouvrages d'architecture [...]. Il faut qu'il sache le dessein, la géometrie, l'optique, l'aritmétique, l'astrologie \& l'histoire ». Pierre Richelet, Dictionnaire françois, contenant généralement tous les mots, tant vieux que nouveaux ..., nouv. éd., Amsterdam, Jean Elzevir, 1706, p. 69. Pour une définition de la profession d'architecte sous l'Ancien Régime, voir Claude Mignot, "Architectes", dans Lucien Bély (dir.), Dictionnaire de l'ancien régime: royaume de France, XVI $-X V I I I^{e}$ siècles, Paris, Presses Universitaires de France, 2002, p. 76-77.

23. BnF, Collection de lettres circulaires émanées des religieuses de la Visitation-Sainte-Marie des différents monastères de France ou à elles adressées de France ou de l'étranger, t. 75.

24. Cité par Robert Triger, «L'église de la Visitation au Mans et son principal architecte, Sœur AnneVictoire Pillon ", Revue historique et archéologique du Maine, t. 53, 1903, p. 225-268. La source n’a pas pu être retrouvée. 


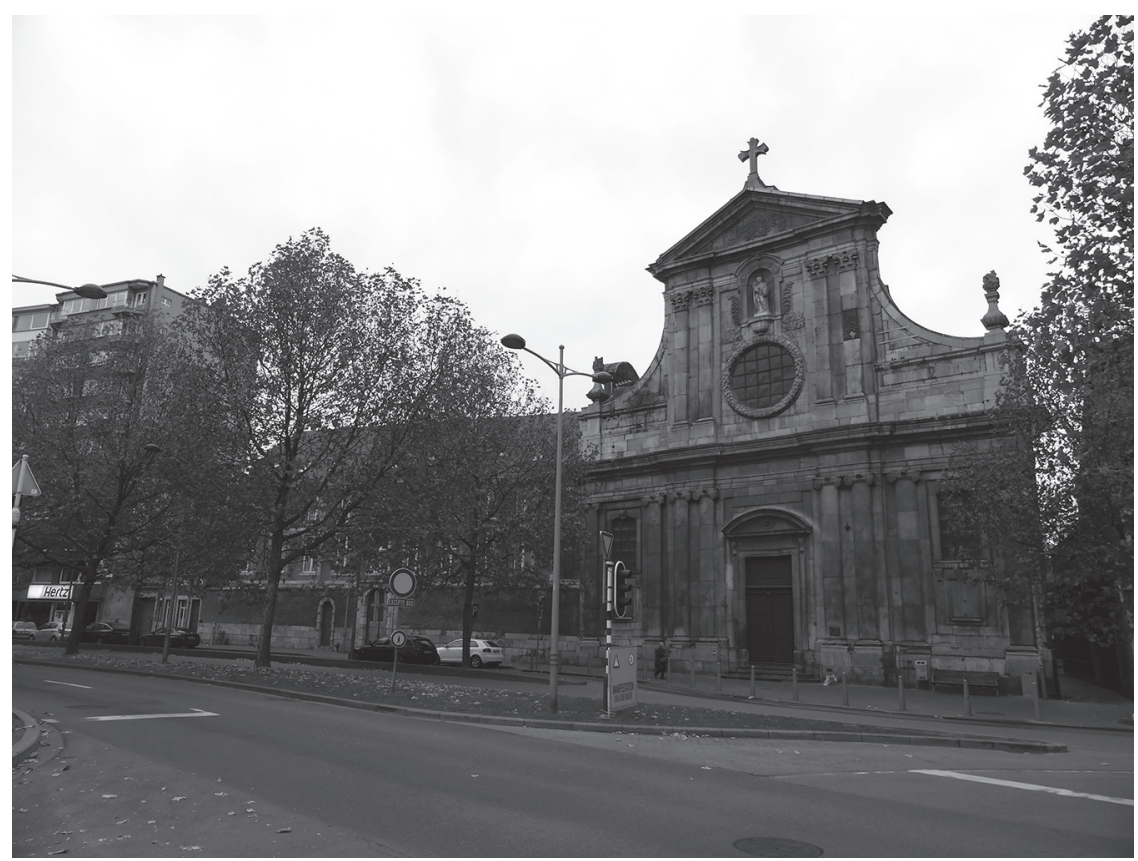

Ill. 3 : Aldegonde Desmoulin (v. 1611-1692), Façade de l'abbatiale de la Paix Notre-Dame à Liège, v. 1680-1690. (C) Cliché Julie Piront.

Enfin, quelques religieuses semblent avoir bénéficié d'un apprentissage dans le cadre familial, avant leur entrée en religion. Bénédictine de la Paix Notre-Dame à Liège, Aldegonde Desmoulin dessine le plan de l'église de la communauté vers 1675 (ill. 3-4). Le registre des professions de la communauté nous renseigne sur son milieu familial : fille d'un peintre de Mons, elle "savoit escrire tout les sorte de lettre en perfection, composer lettre vers, pindre, faire les fleurs à la gomme » ${ }^{25}$ lors de son entrée au couvent. Du dessin à l'architecture, le pas peut être facilement franchi.

\section{Des religieuses sur le terrain: "intendantes " et manouvrières}

Si plusieurs religieuses ont conçu des plans de bâtiments, plus nombreuses sont celles qui ont occupé un rôle concret dans la gestion du chantier et de sa comptabilité. Qualifiées parfois "d'intendantes du bâtiment " ${ }^{26}$, ces femmes occupent

25. Archives des bénédictines de la Paix Notre-Dame à Liège, $7:$ Registre contenant la réception des filles et leur profession, en ce présent monastère de la Paix N[ot]re-Dame, come aussi les dottes qu'elles $\ddot{y}$ ont apportez en aumonne, et les jours de leur trespas commençant l'an 1627, XVII ${ }^{\mathrm{e}}-\mathrm{XVIII} \mathrm{e}^{\mathrm{e}}$ siècles, p. 13 ; Jules Demarteau, op. cit., p. 155.

26. Porrentruy, bibliothèque cantonale du Jura, ms A2598: Annales du monastère de l'annonciade céleste fondé à Porrentruy, XVII ${ }^{\mathrm{e}} \mathrm{XVIII}{ }^{\mathrm{e}}$ siècles, p. 25. 


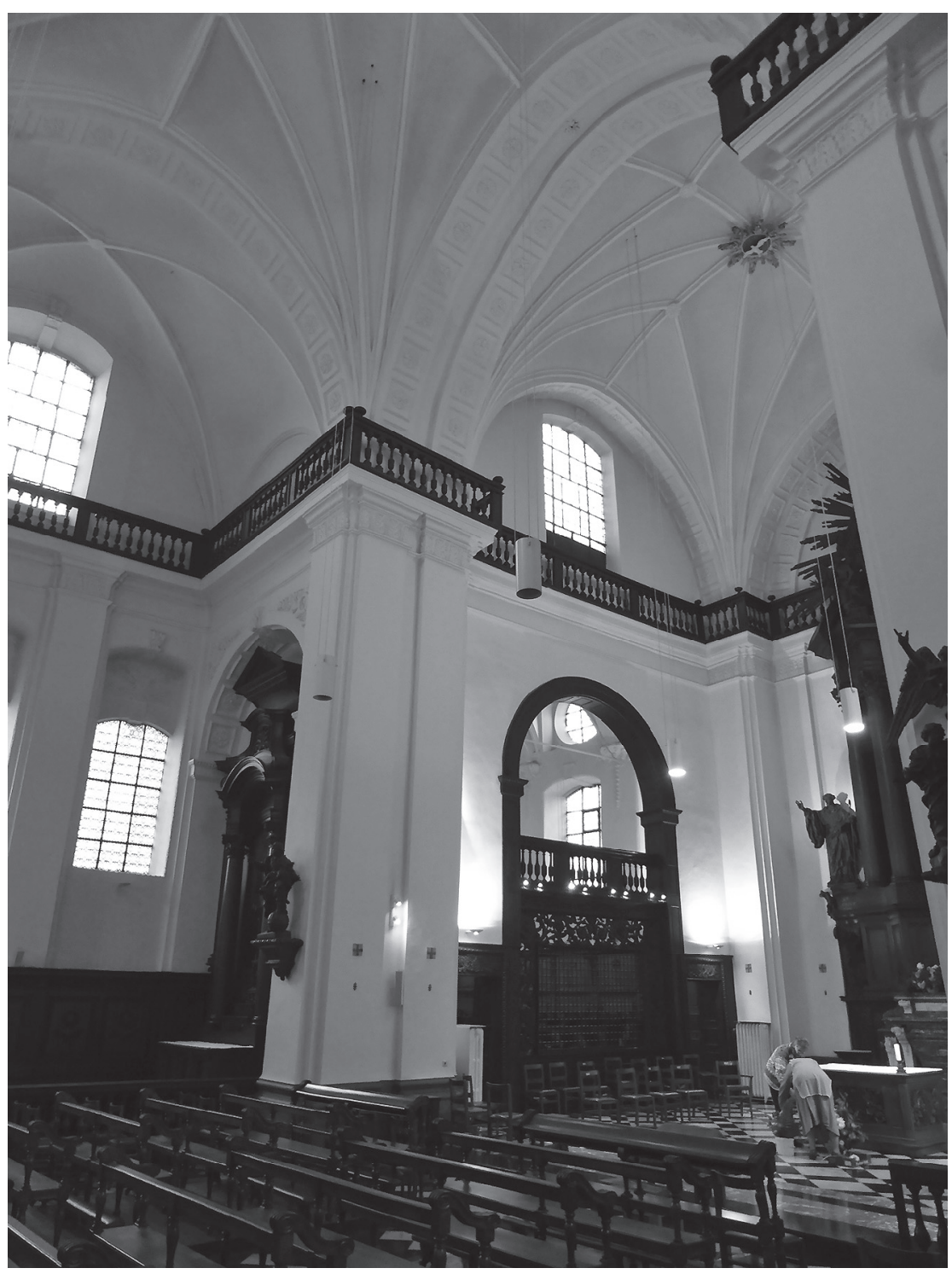

Ill. 4 : Aldegonde Desmoulin (v. 1611-1692), Vue intérieure de l'abbatiale de la Paix Notre-Dame à Liège, v. 1680-1690. (C) Cliché Julie Piront.

souvent conjointement la charge de prieure ou de sous-prieure au sein de la communauté. Ainsi Marie-Élisabeth von Ranzau (v. 1625-1706), fondatrice du monastère des annonciades célestes d'Hildesheim, supervise la construction de l'église, de la sacristie, du chœur des religieuses, de la cuisine, du réfectoire et d'une galerie de communication durant les années 1669-1670: 
On n'a pas icy des entrepreneurs comme en France, il a fallu qu'elle achepte des pierres et du bois, des tuiles, de la terre, de la chaux et payer à part les voitures de toutes ces denrées et puis tous les dimanches venaient 5 ou 6 sortes d'ouvriers demander l'argent de leur semaine, il n'y a eu que le charpentier avec lequel elle a marchandé en gros $[\ldots]^{27}$.

Bien qu'elles soient soumises à une clôture stricte, ces religieuses responsables de chantier communiquent avec les ouvriers pour passer les contrats, donner leurs recommandations, payer les salaires et signer les quittances. Les sources insistent régulièrement sur les compétences de ces femmes, validées par la reconnaissance des hommes de métier. Supérieure des carmélites à Besançon, la mère Thérèse de Jésus Mercier :

commença par évaluer à peu près la dépense sur le plan qu'elle avait dressé [...]. Elle conduisait le bâtiment, donnait ses ordres aux ouvriers, décidait les difficultés qu'ils lui exposaient, leur parlait à chacun dans les termes de leur art, comme un habile architecte. Elle captiva tellement la confiance de ces bonnes gens qu'ils furent heureux, dans tous leurs embarras, de s'adresser à elle ${ }^{28}$.

Malgré les compétences de certaines d'entre elles et le soin qu'elles accordent au choix des entrepreneurs, les communautés ne sont toutefois pas à l'abri des malversations. En 1717, les annonciades célestes de Porrentruy confient à un charpentier le chantier d'une nouvelle aile conventuelle, car son prix et son estimation du temps nécessaire à la construction défient toute concurrence. Dès 1731, le bâtiment commence à s'affaisser et l'expertise en révèle tous les défauts ${ }^{29}$. Les sœurs regrettèrent de n'avoir eu :

une personne destiné à qui on aurois bien gagné de donné une récompense et salaire considérable, pour visiter souvent et estre le plus qu'il fut esté pocible présent, pour voir comme tout ce faisoit, a quoÿ les religieuses ne s'entende rien, et n'on[t] aucune expérience, ne pouvant juger si ses sortes de chose son $[\mathrm{t}]$ bonne et bien faite $[\ldots]^{30}$.

Si les religieuses architectes et les gestionnaires de chantier occupent généralement les fonctions les plus hautes au sein de la communauté, le reste de la communauté n'est pas pour autant exclu de l'entreprise architecturale. La démolition et la préparation du chantier, qui requièrent moins de connaissances et de compétences techniques, peuvent notamment leur être confiées. Les carmélites de Beaune, par exemple, se répartissaient les tâches :

27. Arch. nat. de France, L 1040 (9) : Récit des principaux événements survenus au monastère des annonciades célestes de Hildesheim en 1668-1670, XVII ${ }^{\mathrm{e}}$ siècle, p. 13v.

28. Chroniques de l'ordre des carmélites, op. cit., t. 3, p. 88-89.

29. Porrentruy, bibliothèque cantonale du Jura, ms A2598, p. 73-74 et 82 .

30. Ibid., p. 82. 
Désirant ardemment jouir de leur chère clôture, [elles] se mirent elles-mêmes à murer les portes qui donnaient entrée aux séculiers. Les unes faisaient le mortier, les autres portaient les pierres; deux d'entre elles entreprirent un plancher; et des filles qui avaient été élevées avec délicatesse se chargèrent de ces ouvrages si pénibles" ${ }^{31}$.

La chronique souligne le désir de ces femmes de se conformer à la règle, les érigeant ainsi au rang de modèles à imiter. Comme les travaux d'aiguilles et les tâches domestiques, les besognes des manouvrières viennent mettre en valeur les vertus morales et spirituelles de ces religieuses exemplaires ${ }^{32}$. Cet investissement de la communauté a une finalité économique en épargnant les salaires d'une maind'œuvre. Enfin, troisième motivation et non des moindres : le travail des religieuses réduit le nombre d'ouvriers et la durée de leur présence dans la clôture de la communauté. Florent Boulanger, père récollet et célèbre théoricien de la clôture, préconise de faire entrer les ouvriers à condition que l'ouvrage " ne se puisse faire commodément par les moniales ${ }^{33}$.

L'architecture s'inscrit en somme dans les préoccupations quotidiennes des religieuses aux Temps Modernes. S’ils répondent à des besoins matériels et à la nécessité de vivre sous un toit, les bâtiments monastiques informent aussi sur leur mode de vie. Leurs hauts murs dissimulent les religieuses autant qu'ils donnent à voir au cœur de l'espace urbain. Les religieuses architectes, largement méconnues, mais bien présentes, ont su tirer parti des normes dont elles disposaient et qui participent à la construction identitaire de leurs ordres dans un contexte de multiplication intense des projets religieux féminins. Soumises à la clôture et à un contrôle omniprésent des hommes, ces femmes sont pourtant en contacts permanents avec les corps de métier et leurs sorties s'avèrent possibles pour trouver l'inspiration et superviser les chantiers de leur futur cadre de vie. Les écrits des religieuses révèlent donc une certaine perméabilité de la clôture. Par ailleurs, les compétences revendiquées de ces femmes en matière d'architecture et leur investissement personnel viennent enrichir leurs vertus spirituelles, les érigeant ainsi au rang de modèles exemplaires au sein de la communauté, mais aussi les insérant dans la société urbaine qui les entoure.

Julie PIRONT

chargée de recherches FRS-FNRS université de Liège

31. Chroniques de l'ordre des carmélites, op. cit., t. 3, p. 298.

32. Marc Libert, "Le travail dans les couvents contemplatifs féminins ", Revue belge de philologie et d'histoire, t. 79, fasc. 2, 2001, p. 547-555.

33. Florent Boulanger, Traitez de la closture des religieuses : leur enseignant l'obligation que toutes y ont; Pourquoy elles en peuvent sortir; qui y entrer et avoir accez aux Parloirs, ..., Paris, D. Moreau, 1629 , p. 242. 
